ARTIGO

\title{
PLATAFORMA EXPERIMENTAL PARA ANÁLISE DO RENDIMENTO DE MOTORES DE INDUÇÃO TRIFÁSICOS POR MEIO DE ENSAIOS ESPECÍFICOS RECOMENDADOS NA NORMA TÉCNICA NBR17094-3:2018 ${ }^{1}$
}

\author{
Vinícius Marcos Pinheiro \\ Luciano Coutinho Gomes \\ Cássio Alves de Oliveira
}

\begin{abstract}
RESUMO
Os principais equipamentos encontrados para realização de movimentos mecânicos são os motores elétricos, sendo que a maior parte destes é constituída pelos motores de indução trifásicos. É inegável a crescente preocupação com a questão da eficiência energética, sendo estes equipamentos os maiores consumidores de energia elétrica, principalmente, nas indústrias. Sendo assim, este trabalho apresenta uma plataforma experimental implementada para a obtenção do rendimento dos motores de indução trifásicos a partir da determinação dos parâmetros do circuito equivalente, de ensaios térmicos e ensaios específicos para obter as perdas da respectiva máquina, com base em procedimentos presentes na norma técnica NBR17094-3:2018 da Associação Brasileira de Normas Técnicas. Neste contexto, o rendimento foi obtido para diversos carregamentos para um motor de $3 \mathrm{cv}$ a partir da variação de carga em seu eixo, utilizando, para isso, uma máquina de corrente contínua. Tal plataforma permite verificar o desempenho de motores antigos, motores que passaram por processo de rebobinagem ou motores construídos sob encomenda.
\end{abstract}

Palavras-chaves: NBR17094-3:2018. Motor de indução trifásico. Rendimento. Parâmetros.

\section{INTRODUÇÃO}

Os motores de indução trifásicos (MIT) são máquinas elétricas responsáveis por converter energia elétrica em energia mecânica (FRANCISCO, 2006), com pequena perda de energia. É um equipamento robusto, de funcionamento simples e com pouca necessidade de manutenção (GUEDES, 1994).

Principalmente no meio industrial, o motor de indução trifásico desempenha um importante papel para tornar os processos produtivos mais rápidos e eficientes, em vários

\footnotetext{
${ }^{1}$ Como citar este artigo: PINHEIRO, Vinícius Marcos; GOMES, Luciano Coutinho; OLIVEIRA, Cássio Alves de. Plataforma experimental para análise do rendimento de motores de indução trifásicos por meio de ensaios específicos recomendados na norma técnica NBR17094-3:2018. ForScience: revista científica do IFMG, Formiga, v. 7, n. 2, e00633, jul./dez. 2019. DOI: 10.29069/forscience.2019v7n2.e633.
} 
PINHEIRO, Vinícius Marcos; GOMES, Luciano Coutinho; OLIVEIRA, Cássio Alves de. Plataforma experimental para análise do rendimento de motores de indução trifásicos por meio de ensaios específicos recomendados na norma técnica NBR17094-3:2018

tipos de aplicações, como (GARCIA, 2003): bombas para movimentação de líquidos, ventiladores para gases, compressores, moinhos, misturadores, movimentação de esteiras rolantes, dentre vários outros e, segundo o Ministério de Minas e Energia, tais motores acima mencionados são, no Brasil, "o equipamento que processa a maior parcela de energia elétrica" (MINISTÉRIO DE MINAS E ENERGIA, 2017). Tal fato mostra a importância de estudar o rendimento da respectiva máquina, pois, como é apresentado pela WEG (2009), o uso de motores eficientes pode gerar uma economia elevada, podendo representar mais de $8 \%$ sobre toda energia consumida em uma indústria.

Muitos motores, principalmente os de grande porte, sofrem um processo de recondicionamento, onde as bobinas dos mesmos são substituídas. Um estudo apresentado por Venero (2008) indica que é viável optar por recondicionamento de motores acima de 7,5 cv em vez de realizar sua completa substituição por um novo, como pode ser visualizado por meio da Figura 1.

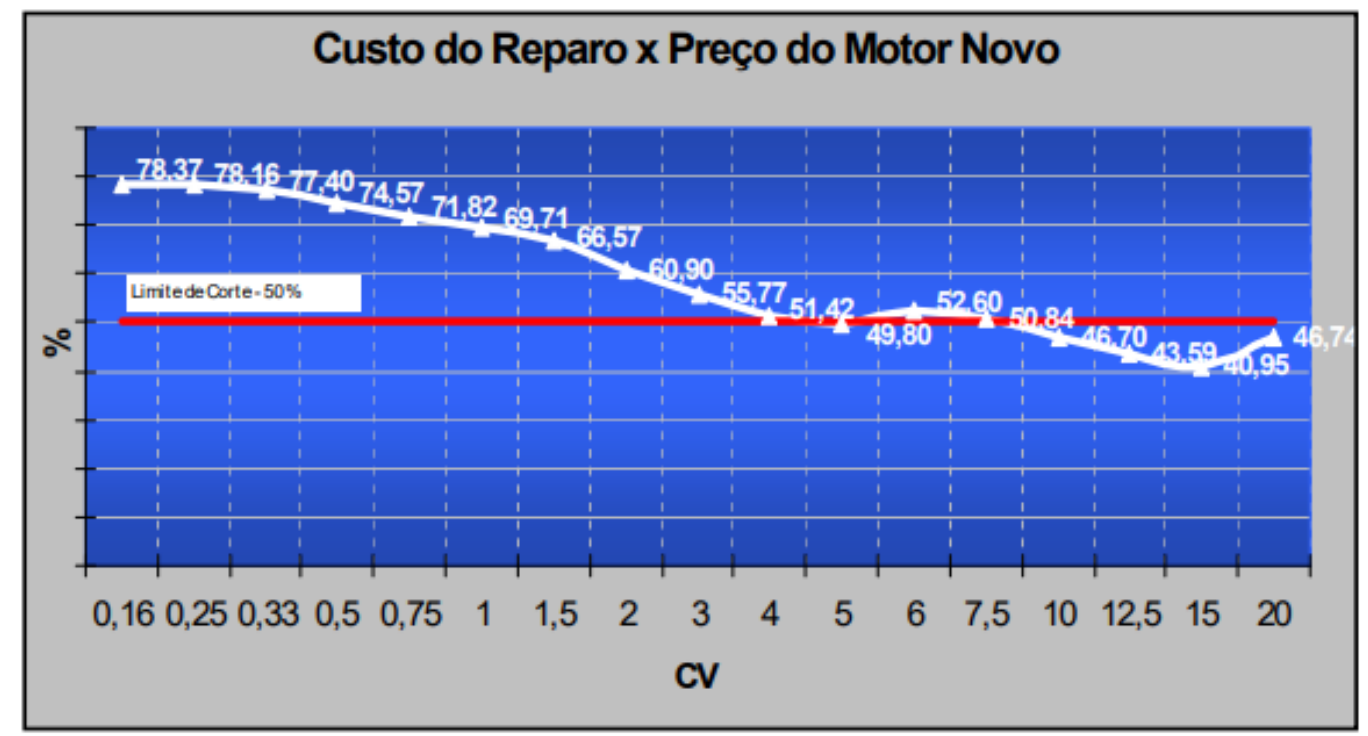

Figura 1 - Custo de reparo x Preço do motor novo Fonte: Venero (2008).

A Figura 1 indica que o custo de reparo para motores acima de 7,5 cv é menor que a metade do custo de aquisição de um respectivo motor novo, sendo melhor optar-se pelo recondicionamento do que pela sua troca. Contudo, depois do recondicionamento, muitas informações a respeito do motor podem ser perdidas ou alteradas, dentre elas, o rendimento, o que acaba impossibilitando um estudo eficaz a respeito do consumo de energia total, mesmo sabendo que este equipamento, principalmente no Brasil, é um dos grandes responsáveis pelo consumo de energia elétrica, como é abordado por De Almeida (2003) e Garcia (2007). 
PINHEIRO, Vinícius Marcos; GOMES, Luciano Coutinho; OLIVEIRA, Cássio Alves de. Plataforma

$\mathrm{Na}$ área de eficiência, pode ser citado o trabalho realizado por Ribeiro (2008), voltado para metodologias de eficiência energética do MIT relacionadas ao controle do mesmo e o estudo de eficiência relacionada com o carregamento imposto ao motor, realizado por Campana et al. (2000).

Assim, este trabalho teve como intuito desenvolver uma plataforma experimental que possa ser empregada para analisar o rendimento de motores de indução trifásicos. Tal como os transformadores possuem ensaios específicos que devem ser realizados a fim de garantir um perfeito funcionamento e, também, estudo de eficiência, como é abordado por Cardoso (2005), gerou a concepção de que o mesmo poderia ser aplicado aos motores de indução trifásicos, de tal forma a contribuir para o estudo de eficiência energética, ação que vem tomando grandes proporções nos últimos anos (CARDOSO et al., 2009).

A plataforma desenvolvida tem como base a norma NBR17094-3:2018, que aborda os ensaios necessários para determinar diversos parâmetros e características, como o próprio rendimento, dos motores de indução trifásicos.

As vantagens da adoção de tal norma estão relacionadas com a padronização da execução dos ensaios, assim como possibilitar a realização dos mesmos de forma adequada, confiável e segura.

\section{MATERIAL E MÉTODOS}

\subsection{Plataforma de ensaios}

A Figura 2 representa o diagrama da plataforma de ensaios com os respectivos equipamentos que a constituem, sendo os mesmos apresentados em seguida. 
PINHEIRO, Vinícius Marcos; GOMES, Luciano Coutinho; OLIVEIRA, Cássio Alves de. Plataforma experimental para análise do rendimento de motores de indução trifásicos por meio de ensaios específicos recomendados na norma técnica NBR17094-3:2018

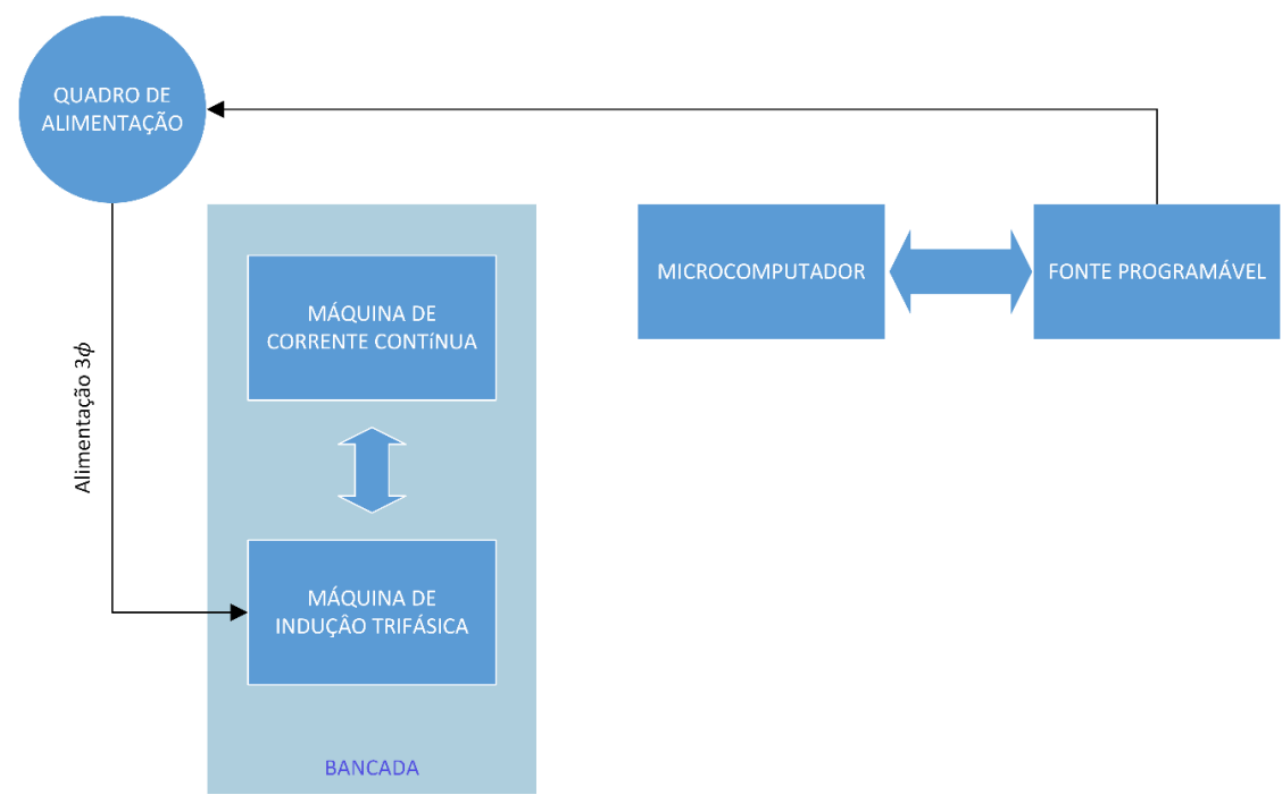

Figura 2 - Diagrama da plataforma de ensaios Fonte: Elaborada pelo autor.

\subsubsection{Bancada experimental}

Para a realização dos ensaios é de suma importância ter uma estrutura na qual se possa efetuar os mesmos de forma adequada. Sendo assim, o laboratório conta com uma bancada experimental desenvolvida para este propósito, que permite ensaios de motores de até $5 \mathrm{cv}$.

A Figura 3 representa o esquema de tal bancada, na qual são realizados os ensaios. Seu projeto foi desenvolvido no SolidWorks, visando a criação de uma estrutura robusta que possibilitasse a realização das atividades de forma prática e segura.

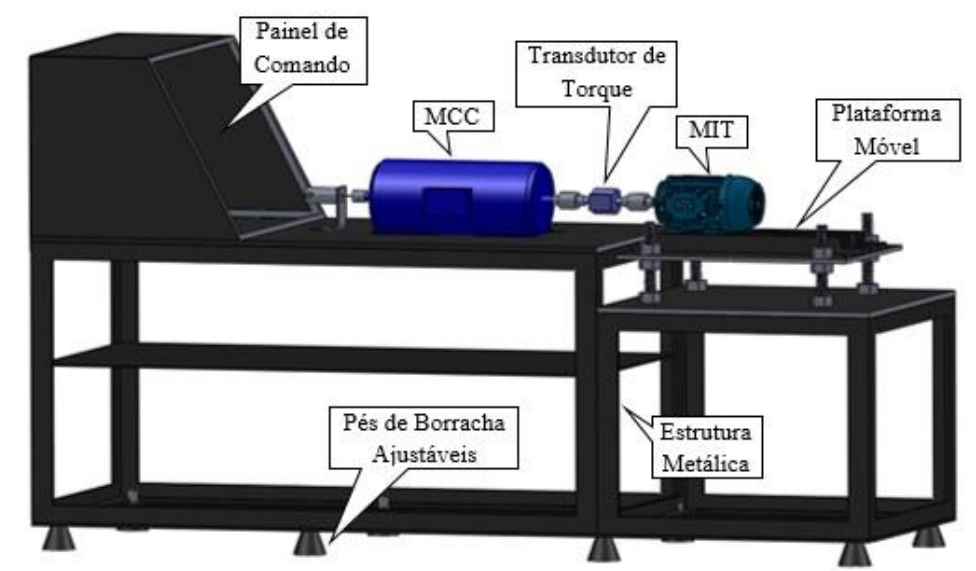

Figura 3 - Representação da bancada experimental Fonte: Elaborada pelo autor. 
PINHEIRO, Vinícius Marcos; GOMES, Luciano Coutinho; OLIVEIRA, Cássio Alves de. Plataforma

Analisando a Figura 3, pode-se pontuar as principais características da bancada que a tornam uma estrutura ideal para a realização dos ensaios: os pés de borracha, além de melhorar com relação ao atrito, por serem antiderrapante, possibilitam um nivelamento adequado com o solo, uma vez que os mesmos são ajustáveis; a plataforma móvel proporciona o adequado acoplamento do eixo do motor em estudo com a máquina Corrente Contínua (CC), visto que é possível ajustá-la para diferentes níveis de altura; a base permite fixar uma série de motores com diferentes tipos de carcaças e é constituída de aço carbono de meia polegada de espessura, atuando justamente na questão da vibração que se origina no funcionamento do motor, tentando reduzi-la de tal forma a não prejudicar os ensaios.

O modelo real da bancada presente no laboratório é apresentado na Figura 4.

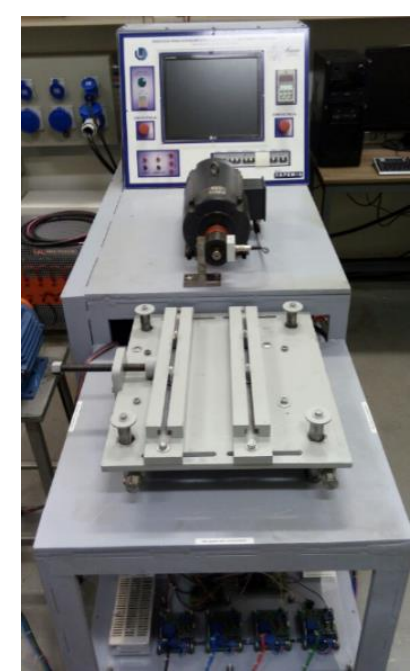

Figura 4 - Bancada experimental

Fonte: Elaborada pelo autor.

\subsubsection{Fonte programável}

A alimentação do motor de indução trifásico, bem como a aquisição das grandezas necessárias no decorrer do estudo, como tensão, corrente, potência e fator de potência, são realizados por meio de uma fonte programável, representada pela Figura 5, cujo modelo é o CSW5550 da fabricante AMETEK Programmable Power. 


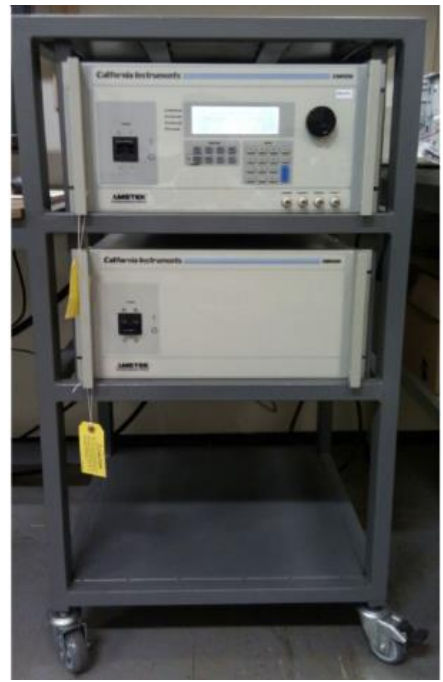

Figura 5 - Fonte programável Fonte: Elaborada pelo autor.

\subsubsection{Máquina de corrente contínua}

Para realizar a imposição de carga ao eixo do motor de indução trifásico é utilizado uma máquina de corrente contínua, representado pela Figura 6.

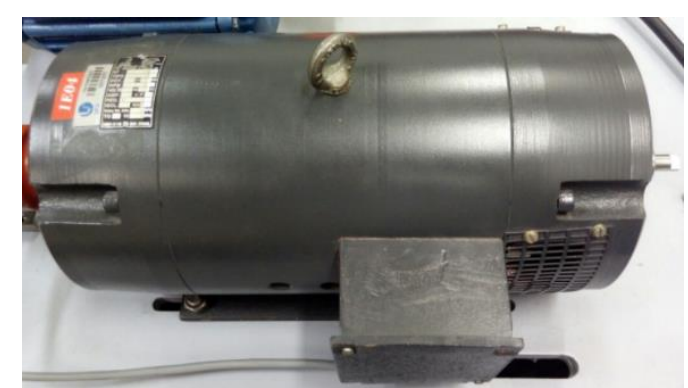

Figura 6 - Máquina de corrente contínua

Fonte: Elaborada pelo autor.

A máquina de corrente contínua é acoplada ao eixo do motor de indução com o simples objetivo de impor qualquer valor de carga ao motor em estudo, tornando possível a análise do rendimento para diversos carregamentos.

\subsubsection{Motor de indução trifásico}

O motor de indução trifásico analisado é da fabricante WEG, cujos dados de placa estão apresentados no Quadro 1. 
PINHEIRO, Vinícius Marcos; GOMES, Luciano Coutinho; OLIVEIRA, Cássio Alves de. Plataforma experimental para análise do rendimento de motores de indução trifásicos por meio de ensaios específicos recomendados na norma técnica NBR17094-3:2018

\begin{tabular}{|c|c|}
\hline \multicolumn{2}{|c|}{$\begin{array}{c}\text { Motor de indução trifásico gaiola de esquilo WEG } \\
\text { Categoria } \mathrm{N}-60 \mathrm{~Hz}-\mathrm{IP} 55-\mathrm{F} . \mathrm{S} 1,15-\mathrm{S} 1-\text { Clas.Isol } \mathrm{F}\end{array}$} \\
\hline Potência $(\mathrm{KW} / \mathrm{CV})$ & $2,2 / 3,0$ \\
\hline Tensão $(\Delta / \mathrm{Y})$ & $220 / 380 \mathrm{~V}$ \\
\hline Corrente $(\Delta / Y)$ & $8,39 / 4,86 \mathrm{~A}$ \\
\hline Fator de Potência & 0,84 \\
\hline Velocidade & $3450 \mathrm{rpm}$ \\
\hline Rendimento & $81,9 \%$ \\
\hline $\begin{array}{c}\text { Razão entre a corrente de partida } \\
\text { e a nominal }\end{array}$ & 6,7 \\
\hline
\end{tabular}

Quadro 1-Dados de placa do motor de indução trifásico utilizado

Fonte: Elaborada pelo autor.

\subsection{Norma NBR17094-3:2018}

A norma brasileira NBR17094-3:2018 trata a respeito de motores de indução trifásicos. Nela estão descritos os ensaios e os cuidados necessários para se obter o rendimento da máquina, sendo que, primeiramente, deve-se determinar os parâmetros do circuito equivalente a partir de três ensaios, os quais são:

- Ensaio para determinação da resistência do enrolamento do estator.

- $\quad$ Ensaio de rotor bloqueado.

- $\quad$ Ensaio a vazio.

Posteriormente é realizado o ensaio térmico para determinar a temperatura especificada, na qual os parâmetros do circuito equivalente deverão estar referenciados para possibilitar a determinação do rendimento. Por fim, é realizado o ensaio onde se varia a carga imposta ao eixo do MIT, cuja finalidade é determinar o rendimento para diferentes valores de carregamento.

\subsection{Circuito equivalente}

Os parâmetros do circuito equivalente do motor de indução trifásico são de suma importância para qualquer estudo acerca da máquina, pois, todo seu equacionamento é em função de tais grandezas. Sendo assim, é importante determiná-las de forma mais exata possível. A NBR17094-3:2018 traz, detalhadamente, como os ensaios para a determinação 
dos parâmetros devem ser realizados. Os parâmetros podem ser observados na Figura 7 a seguir:

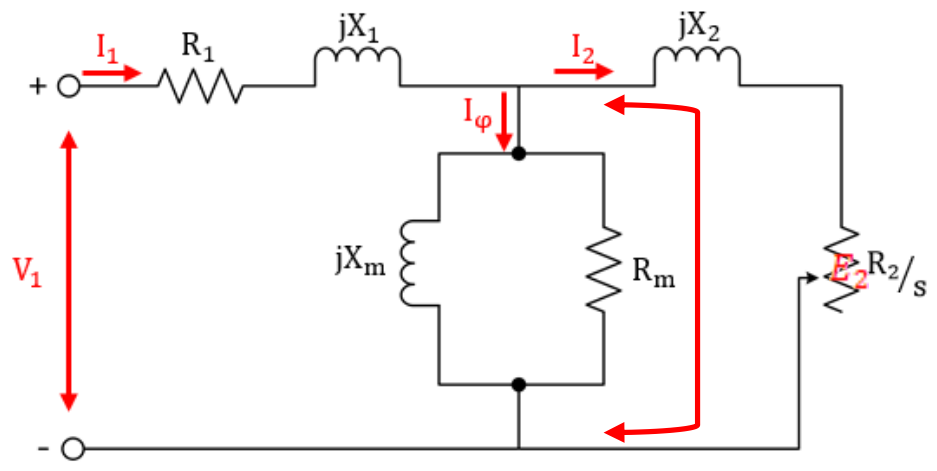

Figura 7 - Circuito equivalente do motor de indução trifásico Fonte: Elaborada pelo autor.

Onde:

$\hat{\mathrm{I}}_{1}-$ Corrente do estator [A].

$V_{1}$ - Tensão no terminal do estator [V].

$X_{1}-$ Reatância de dispersão do estator [ $\left.\Omega\right]$.

$R_{1}-$ Resistência do estator $[\Omega]$.

$R_{2}-$ Resistência do rotor $[\Omega]$.

$X_{2}-$ Reatância de dispersão do rotor [ $\left.\Omega\right]$.

$\tilde{\mathrm{I}}_{\varphi}-$ Corrente de excitação [A].

$\tilde{\mathrm{I}}_{2}-$ Corrente de carga $[\mathrm{A}]$.

$E_{2}-$ Força Contra Eletromotriz [V].

$R_{m}-$ Resistência de perda no núcleo [ $\left.\Omega\right]$.

$X_{m}-$ Reatância de magnetização $[\Omega]$.

$s$ - Escorregamento.

\section{RESULTADOS}

\subsection{Determinação da resistência do enrolamento do estator}


Para determinar a resistência do enrolamento do estator, optou-se pelo método da tensão e corrente descritos na norma NBR17094-3:2018 e, seguindo as recomendações presentes na respectiva norma, obtém-se as medições para cada fase do motor como está representado nas Tabelas 1,2 e 3.

Tabela 1 - Medidas para a fase A

\begin{tabular}{cccc}
\hline \multicolumn{4}{c}{ Circuito da Fase A } \\
\hline $\mathbf{V}(\mathbf{V})$ & $\mathbf{I}(\mathbf{A})$ & $\begin{array}{c}\boldsymbol{R}_{\text {circuito- }} \\
(\mathbf{\Omega})\end{array}$ & $\begin{array}{c}\text { Temperatura } \\
\text { de ensaio }\left({ }^{\circ} \mathbf{C}\right)\end{array}$ \\
\hline 3,76 & 1,72 & 2,186 & 25 \\
\hline 6,60 & 3,02 & 2,185 & 27 \\
\hline 9,18 & 4,14 & 2,217 & 27 \\
\hline
\end{tabular}

Fonte: Elaborada pelo autor.

Tabela 2 - Medidas para a fase B

\begin{tabular}{cccc}
\hline \multicolumn{4}{c}{ Circuito da Fase B } \\
\hline $\mathbf{V}(\mathbf{V})$ & $\mathbf{I}(\mathbf{A})$ & $\begin{array}{c}\boldsymbol{R}_{\text {circuito- }} \\
(\boldsymbol{\Omega})\end{array}$ & $\begin{array}{c}\text { Temperatura } \\
\text { de ensaio }\left({ }^{\circ} \mathbf{C}\right)\end{array}$ \\
\hline 4,06 & 1,86 & 2,182 & 28 \\
\hline 6,63 & 3,02 & 2,195 & 28 \\
\hline 9,19 & 4,16 & 2,209 & 29 \\
\hline
\end{tabular}

Fonte: Elaborada pelo autor.

Tabela 3 - Medidas para a fase C

\begin{tabular}{|c|c|c|c|}
\hline \multicolumn{4}{|c|}{ Circuito da Fase $\mathbf{C}$} \\
\hline $\mathbf{V}(\mathbf{V})$ & I (A) & 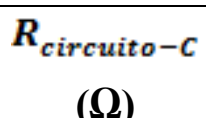 & $\begin{array}{r}\text { Temperatura } \\
\text { de ensaio }\left({ }^{\circ} \mathrm{C}\right)\end{array}$ \\
\hline 4,41 & 1,98 & 2,227 & 30 \\
\hline 6,62 & 2,97 & 2,228 & 32 \\
\hline 9,18 & 4,07 & 2,255 & 36 \\
\hline
\end{tabular}

Fonte: Elaborada pelo autor.

Posteriormente, deve-se realizar a correção das resistências encontradas para uma temperatura padrão, que, segundo a norma, é a temperatura ambiente de $25^{\circ} \mathrm{C}$. Para tal, é empregado a Equação 1 a seguir.

$$
R_{s}=\frac{R_{t}\left(t_{s}+k\right)}{\left(t_{t}+k\right)}
$$

Onde:

$R_{s}-$ Resistência do enrolamento corrigida para uma temperatura especificada, $t_{s}$.

$t_{s}$ - Temperatura especificada para correção da resistência, em graus Celsius. 
PINHEIRO, Vinícius Marcos; GOMES, Luciano Coutinho; OLIVEIRA, Cássio Alves de. Plataforma experimental para análise do rendimento de motores de indução trifásicos por meio de ensaios específicos recomendados na norma técnica NBR17094-3:2018

$R_{t}-$ Resistência do enrolamento obtida no ensaio à temperatura $t_{t}$.

$t_{t}$ - Temperatura do enrolamento no momento da medição da resistência, em graus Celsius.

$k$-Constante baseada no material condutor do enrolamento. (Para cobre eletrolítico com $100 \%$ de condutividade $\mathrm{K}=234,5$ e alumínio com condutividade em volume de $62 \%, \mathrm{~K}=225$ ).

Para efeitos de simplificação, a Tabela 4 indica a resistência média para cada fase à temperatura de $25^{\circ} \mathrm{C}$.

Tabela 4 - Resistência média dos enrolamentos do estator por fase a $25^{\circ} \mathrm{C}$

\begin{tabular}{ccc}
\hline Fase $\mathbf{A}$ & Fase B & Fase C \\
\hline $2,184 \Omega$ & $2,167 \Omega$ & $2,172 \Omega$ \\
\hline
\end{tabular}

Fonte: Elaborada pelo autor.

Logo, a resistência média do enrolamento do estator será dada pela média aritmética dos valores apresentados na Tabela 4, tendo-se um valor igual a:

$$
R_{\text {média }_{\text {estator }}}=\frac{2,184+2,167+2,172}{3}=2,174 \Omega
$$

\subsection{Ensaio de rotor bloqueado}

O ensaio de rotor bloqueado é realizado a fim de determinar a resistência do rotor, assim como as reatâncias de dispersão. Seguindo as recomendações da norma, realiza-se o bloqueio do eixo do motor de indução, como se pode observar na Figura 8.

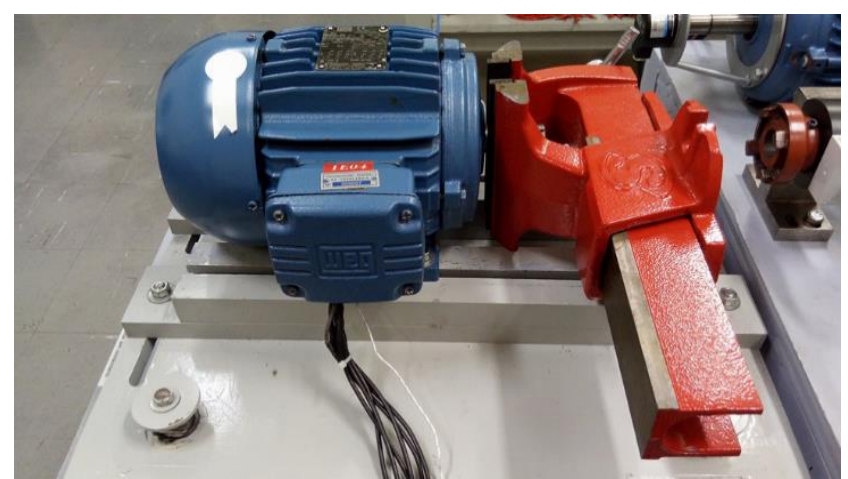

Figura 8 - Bloqueio do eixo do motor de indução Fonte: Elaborada pelo autor. 
PINHEIRO, Vinícius Marcos; GOMES, Luciano Coutinho; OLIVEIRA, Cássio Alves de. Plataforma experimental para análise do rendimento de motores de indução trifásicos por meio de ensaios específicos recomendados na norma técnica NBR17094-3:2018

Posteriormente, é aplicado tensão alternada reduzida nos terminais do MIT, de tal forma a se obter corrente nominal. A NBR17094-3:2018 recomenda que o ensaio seja realizado o mais rápido possível para não danificar os enrolamentos do motor.

Na Tabela 5 estão apresentadas as medições realizadas para este ensaio.

Tabela 5 - Medições para o ensaio de rotor bloqueado via fonte

\begin{tabular}{cccc}
\hline Grandeza medida & FASE A & FASE B & FASE C \\
\hline Tensão (Vrms) & 43,3 & 43,3 & 43,3 \\
\hline Corrente (Arms) & 8,39 & 8,59 & 8,58 \\
\hline Fase $(\boldsymbol{\phi})$ & 0 & 240,3 & 120,5 \\
\hline Potência (kW) & 0,1 & 0,11 & 0,1 \\
\hline $\begin{array}{c}\text { Potência Aparente } \\
\text { (kVA) }\end{array}$ & 0,21 & 0,215 & 0,214 \\
\hline Fator de Potência & 0,49 & 0,5 & 0,48 \\
\hline Temperatura & & $36^{\circ} \mathrm{C}$ & \\
\hline
\end{tabular}

Fonte: Elaborada pelo autor.

A Tabela 6 apresenta os valores dos parâmetros do motor, já supracitados.

Tabela 6 - Parâmetros calculados

\begin{tabular}{cc}
\hline Parâmetro & Valor $(\mathbf{\Omega})$ \\
\hline $\begin{array}{c}\text { Resistência do enrolamento } \\
\text { do rotor }\end{array}$ & 2,004 \\
\hline $\begin{array}{c}\text { Reatância de dispersão do } \\
\text { estator }\end{array}$ & 3,845 \\
\hline $\begin{array}{c}\text { Reatância de dispersão do } \\
\text { rotor }\end{array}$ & 3,845 \\
\hline
\end{tabular}

Fonte: Elaborada pelo autor.

\subsection{Ensaio a vazio}

O ensaio a vazio possui como finalidade a determinação da resistência de perdas no núcleo, a reatância de magnetização e as perdas a vazio. As medições para este ensaio são realizadas seguindo os procedimentos descritos pela NBR17094-3:2018, tomando-se os cuidados abordados na mesma. O respectivo ensaio é realizado com o eixo do motor de indução trifásico totalmente livre. $\mathrm{Na}$ Tabela 7 estão indicados os valores das medições efetuadas. 
PINHEIRO, Vinícius Marcos; GOMES, Luciano Coutinho; OLIVEIRA, Cássio Alves de. Plataforma experimental para análise do rendimento de motores de indução trifásicos por meio de ensaios específicos recomendados na norma técnica NBR17094-3:2018

Tabela 7 - Medições obtidas durante o ensaio a vazio via fonte

\begin{tabular}{cccc}
\hline Grandeza medida & FASE A & FASE B & FASE C \\
\hline Tensão (Vrms) & 219,97 & 219,97 & 219,97 \\
\hline Corrente (Arms) & 4,31 & 4,43 & 4,31 \\
\hline Potência (kW) & 0,08 & 0,08 & 0,07 \\
\hline Fator de Potência & 0,15 & 0,14 & 0,13 \\
\hline Temperatura & & $48^{\circ} \mathrm{C}$ & \\
\hline
\end{tabular}

Fonte: Elaborada pelo autor.

Com os valores das medições em mãos e, seguindo os procedimentos dos cálculos presentes na norma, obtém-se os valores dos parâmetros desejados, conforme apresentados na Tabela 8.

Tabela 8 - Parâmetros calculados

\begin{tabular}{cc}
\hline Parâmetro & Valor $(\mathbf{\Omega})$ \\
\hline$R_{m}$ & 593,491 \\
\hline$X_{m}$ & 83,924 \\
\hline
\end{tabular}

Fonte: Elaborada pelo autor.

A potência de entrada total medida, também conhecida como perdas a vazio, representa a soma das perdas do estator, de atrito/ventilação e do núcleo. Sendo assim, tem-se que:

$$
P_{\circ}=I^{2} R_{1}+P_{A V}+P_{N}
$$

Onde:

$P_{o}-$ Perdas a vazio.

$I^{2} R_{1}$ - Perdas joulicas no estator, sendo $R_{1}$ a resistência corrigida para a temperatura do ensaio a vazio.

$P_{A V}-$ Perdas por atrito/ventilação.

$P_{N}-$ Perdas no núcleo.

O somatório das potências de cada fase obtidas durante o ensaio a vazio, apresentadas na Tabela 7 , fornece o total de perdas a vazio $\left(P_{o}\right)$. Realizando o somatório de todas elas, temse um valor de:

$$
P_{\mathrm{o}}=(0,08+0,08+0,07) \times 1000=230 \mathrm{~W}
$$


PINHEIRO, Vinícius Marcos; GOMES, Luciano Coutinho; OLIVEIRA, Cássio Alves de. Plataforma experimental para análise do rendimento de motores de indução trifásicos por meio de ensaios específicos recomendados na norma técnica NBR17094-3:2018

Para o cálculo das perdas no estator, o primeiro passo é realizar a correção da resistência do estator para a temperatura do presente ensaio, que é de $48^{\circ} \mathrm{C}$. De posse da resistência do enrolamento do estator corrigida, pode-se realizar, portanto, o cálculo da perda sobre o mesmo, utilizando a média das correntes presente da Tabela 7. Logo, tem-se que:

$$
R_{1_{48^{8} \mathrm{C}}} I_{\text {med }}^{2}=3 \times 2,366 \times(4,35 / \sqrt{3})^{2}=44,754 \mathrm{~W}
$$

Para a determinação das perdas por atrito/ventilação e as perdas no núcleo, deve-se adotar o seguinte procedimento recomendado pela norma: segundo a NBR17094-3:2018, a separação da perda do núcleo, da perda por atrito/ventilação, deve ser realizada pela leitura de tensão, corrente e potência de entrada à frequência nominal, variando a tensão de $125 \%$ da tensão nominal até o ponto onde o decréscimo de tensão provoque um aumento de corrente.

Depois de realizado o procedimento acima, por meio da variação de tensão e aquisição dos valores via fonte, obteve-se o seguinte ponto, presente na Tabela 9.

Tabela 9 - Medições obtidas para a separação das perdas por atrito/ventilação e a núcleo via fonte

\begin{tabular}{cccc}
\hline Grandeza medida & FASE A & FASE B & FASE C \\
\hline Tensão (Vrms) & 51,94 & 51,94 & 51,93 \\
\hline Corrente (Arms) & 1,27 & 1,29 & 1,26 \\
\hline Potência (kW) & 0,03 & 0,03 & 0,03 \\
\hline Fator de Potência & 0,7 & 0,69 & 0,69 \\
\hline Fonte Elarora
\end{tabular}

Fonte: Elaborada pelo autor.

O somatório das potências de cada fase da Tabela 9 fornece a perda por atrito /ventilação. Logo:

$$
P_{A V}=(0,03+0,03+0,03) \times 1000=90 \mathrm{~W}
$$

Substituindo, assim, os valores das perdas já calculadas na Equação 2, pode-se, então, determinar o valor das perdas no núcleo.

$$
P_{N}=P_{\circ}-I^{2} R-P_{A V}=230-44,754-90=95,246 \mathrm{~W}
$$

\subsection{Ensaio Térmico}

O ensaio térmico tem como objetivo determinar a elevação de temperatura de determinadas partes do motor acima da temperatura ambiente, quando o mesmo está funcionando sob condição de uma carga especificada.

Para a realização de tal ensaio, é necessário impor carga ao eixo do motor seguindo as recomendações presentes na norma NBR17094-3:2018 e para tal, é empregado uma máquina 
PINHEIRO, Vinícius Marcos; GOMES, Luciano Coutinho; OLIVEIRA, Cássio Alves de. Plataforma experimental para análise do rendimento de motores de indução trifásicos por meio de ensaios específicos recomendados na norma técnica NBR17094-3:2018

de corrente contínua, onde a mesma é acoplada diretamente ao eixo do motor de indução trifásico, como pode ser visualizado na Figura 9.

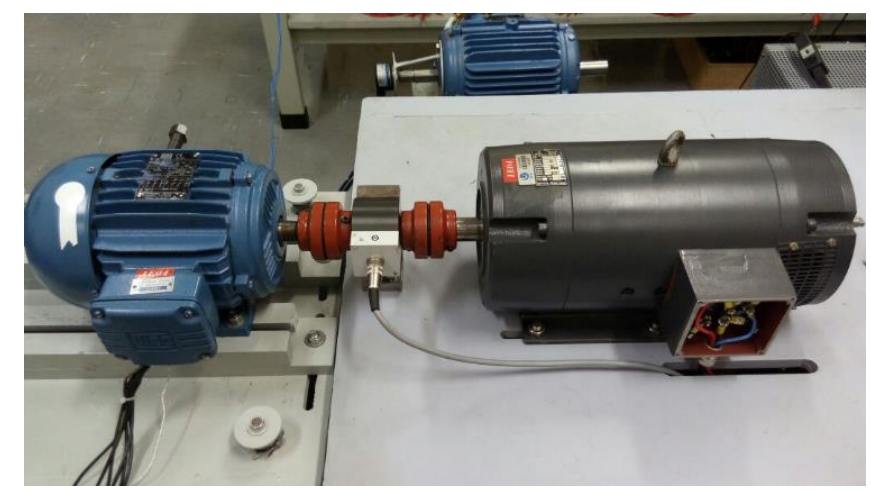

Figura 9 - Acoplamento entre o MIT e a máquina CC

Fonte: Elaborada pelo autor.

$\mathrm{Na}$ Tabela 10 estão apresentados os valores das medições realizadas, em que foi aplicado uma carga de tal forma que o motor operasse na condição do fator de serviço, como é recomendado pela respectiva norma.

Tabela 10 - Medição realizada para determinação da elevação de temperatura através do enrolamento do estator

Fator de serviço

Corrente nominal

(A)
Corrente média medida durante $o$ ensaio (A) 
PINHEIRO, Vinícius Marcos; GOMES, Luciano Coutinho; OLIVEIRA, Cássio Alves de. Plataforma experimental para análise do rendimento de motores de indução trifásicos por meio de ensaios específicos recomendados na norma técnica NBR17094-3:2018

\subsection{Ensaio para determinação do rendimento}

O rendimento em motores elétricos é responsável por indicar o quanto de energia elétrica está sendo convertida em energia eletromecânica (DEL TORO, 1994). A Equação 3 indica uma forma genérica de se calcular o rendimento onde a potência de saída é igual a potência de entrada menos as perdas presentes no motor.

$$
\text { Rendimento }(\mathrm{n})=\frac{\text { Potência de saída }}{\text { Potência de entrada }}
$$

A NBR17094-3:2018 estabelece dez métodos para a determinação do rendimento, dentre os quais, foi optado pelo seguinte para a presente análise:

- Medição da potência elétrica sob carga com segregação e valor assumido das perdas suplementares.

Tal método consiste na medição da potência de entrada, sendo a potência de saída obtida subtraindo-se as perdas da primeira.

As medidas de tensão, corrente, potência de entrada e frequência, presentes na Tabela 12, foram obtidas por meio da fonte programável. A temperatura ambiente foi obtida através de um termopar, enquanto que, para medir a velocidade, foi utilizado um tacômetro digital. Para determinar a temperatura do enrolamento do estator foi utilizado a técnica da elevação de temperatura, descrita na respectiva norma. O escorregamento é obtido através da Equação 4 (FITZGERALD, 2006).

$$
S=\frac{N s-N r}{N s} \times 100
$$

Onde:

$S-$ Escorregamento percentual.

$\mathrm{Nr}$ - Velocidade do rotor (rpm).

Ns - Velocidade síncrona (rpm). 
PINHEIRO, Vinícius Marcos; GOMES, Luciano Coutinho; OLIVEIRA, Cássio Alves de. Plataforma experimental para análise do rendimento de motores de indução trifásicos por meio de ensaios específicos recomendados na norma técnica NBR17094-3:2018

Tabela 12 - Medições realizadas para a determinação do rendimento

\section{Carga com relação a nominal}

\begin{tabular}{|c|c|c|c|c|c|}
\hline Descrição & $140 \%$ & $125 \%$ & $100 \%$ & $75 \%$ & $50 \%$ \\
\hline $\begin{array}{l}\text { Temperatura ambiente } \\
\left({ }^{\circ} \mathrm{C}\right)\end{array}$ & 25 & 25 & 25 & 25 & 25 \\
\hline Resistência do rotor $(\Omega)$ & 3,75 & 3,45 & 3,15 & 2,85 & 2,55 \\
\hline $\begin{array}{c}\text { Temperatura do } \\
\text { enrolamento do estator } \\
\left({ }^{\circ} \mathrm{C}\right)\end{array}$ & 213,119 & 177,310 & 141,50 & 105,69 & 69,89 \\
\hline Frequência $(\mathrm{Hz})$ & 60,01 & 60,00 & 60,01 & 60,01 & 60 \\
\hline Velocidade (rpm) & 3275 & 3340 & 3432 & 3508 & 3564 \\
\hline Escorregamento (\%) & 9,028 & 7,222 & 4,667 & 2,555 & 1,010 \\
\hline Tensão de linha (V) & 126,99 & 126,99 & 126,99 & 126,99 & 126,99 \\
\hline Corrente de linha (A) & 11,356 & 10,530 & 8,738 & 6,613 & 4,908 \\
\hline Potência de entrada (W) & 3803 & 3442 & 2732 & 1803 & 853 \\
\hline
\end{tabular}

Fonte: Elaborada pelo autor.

De posse dos parâmetros mensurados, deve-se corrigir o escorregamento para a temperatura especificada do estator, que é $127,317^{\circ} \mathrm{C}$, empregando-se a Equação 5. Os resultados de tal correção estão apresentados na Tabela 13.

$$
S_{s}=\frac{S_{t}\left(t_{s}+k\right)}{\left(t_{t}+k\right)}
$$

Onde:

$S_{s}$ - Escorregamento corrigido para a temperatura especificada do estator, $t_{s}$.

$S_{t}-$ Escorregamento calculado durante o ensaio com carga, na temperatura medida, $t_{t}$.

$t_{s}-$ Temperatura especificada para correção da resistência, em graus Celsius.

$t_{t}$ - Temperatura do enrolamento do estator medida durante o ensaio com carga, em graus

Celsius.

Tabela 13 - Escorregamento corrigido para a temperatura especificada do estator

\section{Carga com relação a nominal}

\begin{tabular}{cccccc}
\hline Descrição & $\mathbf{1 4 0 \%}$ & $\mathbf{1 2 5 \%}$ & $\mathbf{1 0 0 \%}$ & $\mathbf{7 5 \%}$ & $\mathbf{5 0 \%}$ \\
\hline $\begin{array}{c}\text { Escorregamento corrigido } \\
(\%)\end{array}$ & 7,297 & 6,345 & 4,490 & 2,717 & 1,200 \\
\hline
\end{tabular}

Fonte: Elaborada pelo autor. 
O próximo passo é realizar o cálculo das perdas, as quais podem ser resumidas em:

- Perdas no estator.

- Perdas no rotor.

- Perdas no núcleo.

- Perdas por atrito e ventilação.

- Perdas suplementar.

As perdas no núcleo e as perdas por atrito e ventilação são aquelas que foram obtidas no ensaio a vazio. Para o cálculo das perdas no estator é necessário, primeiramente, corrigir a respectiva resistência para a temperatura especificada e, posteriormente, utilizar a Equação 6.

$$
\text { Perda no estator }=I^{2} R_{1}
$$

Para determinar as perdas no rotor, deve-se obter a potência através do entreferro, utilizando a Equação 7.

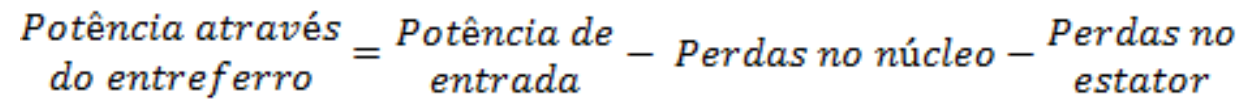

Sendo assim, obtida a potência através do entreferro, pode-se determinar as perdas no rotor pela Equação 8.

$$
\text { Perda no rotor }=\begin{aligned}
& \text { Potência através } \\
& \text { do entreferro }
\end{aligned}
$$

A última perda a ser determinada é a perda suplementar. No método adotado, a perda suplementar nominal é calculada através de valores assumidos, apresentados no Quadro 2.

\begin{tabular}{|c|c|}
\hline Potência nominal do motor & $\begin{array}{c}\text { Perda suplementar-Porcentagem da } \\
\text { potência de saída nominal }\end{array}$ \\
\hline $0,75-90 \mathrm{~kW} \quad 1-125 \mathrm{cv}$ & 1,8 \\
\hline $91-375 \mathrm{~kW} \quad 126-500 \mathrm{cv}$ & 1,5 \\
\hline $376-1839 \mathrm{~kW} 501-2499 \mathrm{cv}$ & 1,2 \\
\hline $1840 \mathrm{~kW}$ e acima $2500 \mathrm{cv}$ & 0,9 \\
\hline
\end{tabular}

Quadro 2 - Valores assumidos da perda suplementar

Fonte: NBR17094-3:2018

A partir da Equação 9 pode-se determinar o valor da perda suplementar nominal $\left(W_{L L}^{\prime}\right)$ utilizando como auxílio os dados do Quadro 2.

$$
W_{L L}^{\prime}=\begin{array}{cc}
\text { Potência nominal } & \text { Porcentagem da potência } \\
\text { do motor } & \text { de saída nominal }
\end{array}
$$

Sendo a potência nominal do motor igual a $2,2 \mathrm{~kW}$, substitui-se os respectivos valores na Equação 9, tendo o valor da perda suplementar nominal. 
PINHEIRO, Vinícius Marcos; GOMES, Luciano Coutinho; OLIVEIRA, Cássio Alves de. Plataforma experimental para análise do rendimento de motores de indução trifásicos por meio de ensaios específicos recomendados na norma técnica NBR17094-3:2018

$$
W_{L L}^{\prime}=2200 x \frac{1,8}{100}=39,6 \mathrm{~W}
$$

Posteriormente, é necessário realizar a correção da perda suplementar para cada valor de carga imposta. Para isto, utiliza-se a Equação 10.

$$
W_{L L}=W_{L L}^{\prime} x\left(\frac{I_{2}}{I_{2}^{\prime}}\right)^{2}
$$

Onde:

$W_{L L}$ - Perda suplementar corrigida.

$I_{2}-$ É o valor da corrente do rotor referente ao ponto da carga para o qual a perda suplementar é para ser determinada.

$I_{2}^{\prime}-$ É o valor da corrente do rotor correspondente à carga nominal.

A corrente no rotor pode ser determinada através da Equação 11.

Onde:

$$
I_{2}=\sqrt{\left(I^{2}-I_{0}^{2}\right)}
$$

$I-\mathrm{O}$ valor da corrente no estator.

$I_{\circ}-\mathrm{O}$ valor de corrente a vazio.

De posse de todas as perdas, pode-se determinar a potência de saída através da Equação 12.

$$
\text { Potência de saída }=\text { Potência de entrada }-\begin{gathered}
\text { Somade todas } \\
\text { as perdas }
\end{gathered}
$$

A Tabela 14 resume todas as perdas calculadas, bem como a potência de saída, que será utilizada para o cálculo do rendimento.

Tabela 14 - Perdas calculadas do motor de indução trifásico estudado (continua)

\begin{tabular}{cccccc}
\hline & \multicolumn{5}{c}{ Carga com relação a nominal } \\
\hline Descrição & $\mathbf{1 4 0 \%}$ & $\mathbf{1 2 5 \%}$ & $\mathbf{1 0 0 \%}$ & $\mathbf{7 5 \%}$ & $\mathbf{5 0 \%}$ \\
\hline Perdas no núcleo $(\mathrm{W})$ & 95,25 & 95,246 & 95,246 & 95,246 & 95,246 \\
\hline $\begin{array}{c}\text { Perdas no estator }(\mathrm{W}) \\
\text { Potência através do } \\
\text { entreferro (W) }\end{array}$ & 390,87 & 336,08 & 231,42 & 132,55 & 73,01 \\
\hline Perdas no rotor (W) & 242,03 & 191,03 & 107,99 & 42,79 & 8,22 \\
\hline $\begin{array}{c}\text { Perdas por atrito e } \\
\text { ventilação (W) }\end{array}$ & 90 & 90 & 90 & 90 & 90 \\
\hline
\end{tabular}

Fonte: Elaborada pelo autor. 
PINHEIRO, Vinícius Marcos; GOMES, Luciano Coutinho; OLIVEIRA, Cássio Alves de. Plataforma experimental para análise do rendimento de motores de indução trifásicos por meio de ensaios específicos recomendados na norma técnica NBR17094-3:2018

Tabela 14 - Perdas calculadas do motor de indução trifásico estudado (continuação)

Carga com relação a nominal

\begin{tabular}{cccccc}
\hline Descrição & $\mathbf{1 4 0 \%}$ & $\mathbf{1 2 5 \%}$ & $\mathbf{1 0 0 \%}$ & $\mathbf{7 5 \%}$ & $\mathbf{5 0 \%}$ \\
\hline Corrente do rotor (A) & 10,49 & 9,59 & 7,58 & 6,61 & 2,27 \\
\hline Perdas suplementares (W) & 84,51 & 70,75 & 44,19 & 19,09 & 3,97 \\
\hline Perdas totais $(\mathrm{W})$ & 836,71 & 693,10 & 568,86 & 379,68 & 270,45 \\
\hline Potência de saída (W) & 2900,34 & 2658,89 & 2163,14 & 1423,32 & 582,55 \\
\hline
\end{tabular}

Fonte: Elaborada pelo autor.

Substituindo os respectivos valores na Equação 3, pode-se, finalmente, determinar o valor do rendimento para cada ponto de carga, cujos valores estão apresentados na Tabela 15 .

Tabela 15 - Determinação do rendimento para cada ponto de carga

\section{Carga com relação a nominal}

\begin{tabular}{cccccc}
\hline Descrição & $\mathbf{1 4 0 \%}$ & $\mathbf{1 2 5 \%}$ & $\mathbf{1 0 0 \%}$ & $\mathbf{7 5 \%}$ & $\mathbf{5 0 \%}$ \\
\hline Rendimento (\%) & 76,26 & 77,25 & 79,18 & 78,94 & 68,29 \\
\hline Fonte: Elaborada pelo autor & & &
\end{tabular}

\section{CONCLUSÃO}

É inegável que a preocupação com a questão da eficiência energética vem tomando grandes proporções nos últimos anos, desde a economia com energia elétrica, como a reutilização de equipamentos que, indiretamente, também contribui nesta ação. Sendo assim, este trabalho veio com o objetivo de contribuir de forma significativa para análise da eficiência de um equipamento que é amplamente utilizado, principalmente, nas indústrias: o motor de indução trifásico.

A construção de uma plataforma para a realização dos ensaios contribui expressivamente para a obtenção dos parâmetros do motor, dado que, na maioria dos casos de recondicionamento, estes podem ser alterados ou perdidos, por não haver, justamente, um meio para obtenção dos mesmos. Além disso, a plataforma permite analisar o comportamento da máquina frente a diversos comportamentos de carga.

Como resultados, foram obtidos no presente trabalho os parâmetros do circuito equivalente de um motor de indução trifásico de $3 \mathrm{cv}$, assim como o seu rendimento para diversos valores de carregamento. 
PINHEIRO, Vinícius Marcos; GOMES, Luciano Coutinho; OLIVEIRA, Cássio Alves de. Plataforma experimental para análise do rendimento de motores de indução trifásicos por meio de ensaios específicos recomendados na norma técnica NBR17094-3:2018

Os ensaios foram realizados de forma segura, sem causar dano ao motor analisado ou às pessoas envolvidas no trabalho. Todos os aspectos presentes na norma NBR17094-3:2018 foram completamente seguidos a fim de obter resultados satisfatórios.

\title{
EXPERIMENTAL PLATFORM FOR EFFICIENCY ANALYSIS OF THE THREE- PHASE INDUCTION MOTORS THROUGH SPECIFIC TESTS RECOMMENDED IN THE TECHNICAL STANDARD NBR17094-3:2018
}

\begin{abstract}
The main equipment used to perform mechanical movements are electric motors, since its majority are composed by three-phase induction motors. There is an undeniable growing concern about the energy saving, being this equipment the major consumers of electricity, especially in industries. Thus, this work presents an experimental platform, implemented to get the performance of the three-phase induction motors from the determination of equivalent circuit tests, thermal tests and specific tests to obtain the losses of the testing machine based on procedures in technical standard NBR17094-3: 2018 of the Brazilian Association of Technical Standards. In this context, the yield was obtained for several loads for a $3 \mathrm{cv}$ engine from the load variation on its axle using a direct current machine. Such platform makes it possible to check the performance of older engines, engines undergoing the rewinding process or custom manufacturing engines.
\end{abstract}

Keywords: NBR17094-3:2018. Three phase induction motor. Efficiency. Parameters.

\section{REFERÊNCIAS}

ASSOCIAÇÃO BRASILEIRA DE NORMAS TÉCNICAS. NBR17094-3:2018: Máquinas elétricas girantes. Parte 3: Motores de indução trifásicos - Métodos de ensaio. Rio de Janeiro, 2018.

CAMPANA, Saulo; OLIVEIRA FILHO, Delly; SOARES, Antônio Alves; OLIVEIRA, Rubens Alves de. Estudo comparativo de métodos para estimativa do carregamento e rendimento de motores elétricos para auditoria energética. Revista Brasileira de Engenharia Agrícola e Ambiental, Campina Grande, v. 4, n. 2, p. 241-246, 2000.

CARDOSO, Bruno Pereira. Eficiência de transformadores de média tensão. 2005. 97 f. Dissertação (Mestrado em Engenharia Elétrica) - Universidade Federal de Itajubá, Itajubá, 2005.

CARDOSO, Rafael Balbino; NOGUEIRA, Luiz Augusto Horta; BORTONI, Edson da Costa; HADDAD, Jamil; SOUZA, Edson Palhares de. Avaliação da economia de energia, atribuída a ações de etiquetagem energética, em motores de indução no Brasil. Revista Brasileira de Energia, São Paulo, v. 15, n. 1, p. 29-47, 2009. 
PINHEIRO, Vinícius Marcos; GOMES, Luciano Coutinho; OLIVEIRA, Cássio Alves de. Plataforma experimental para análise do rendimento de motores de indução trifásicos por meio de ensaios específicos recomendados na norma técnica NBR17094-3:2018

DE ALMEIDA, Anibal T. Market transformation of energy-efficient motor technologies in the EU. Energy Policy, Elsevier, v. 31, n. 6, p. 563-575, 2003.

DEL TORO, Vincent. Fundamentos de máquinas elétricas. Prentice-Hall do Brasil, 1994.

FITZGERALD, Arthur Eugene. Máquinas elétricas: com introdução a eletrônica de potência. 6. ed. Porto Alegre: Bookman, 2006.

FRANCISCO, Antônio. Motores de indução trifásicos. 2006. Disponível em http://www.estgv.ipv.pt/PaginasPessoais/lpestana/maquinas\%20el\%C3\%A9ctricas\%202/aula s\%20te\%C3\%B3ricas/Motores_inducao_tri.pdf. Acesso em: 20 set. 2019.

GARCIA, Agenor Gomes Pinto. Impacto da lei de eficiência energética para motores elétricos no potencial de conservação de energia na indústria. 2003. 139 f. Dissertação. (Mestrado em Planejamento Energético) - Universidade Federal do Rio de Janeiro, Rio de Janeiro, 2003.

GARCIA, Agenor Gomes Pinto. Energy-efficiency standards for electric motors in Brazilian industry. Energy Policy, Elsevier, v. 35, n. 6, p. 3424-3439, 2007.

GUEDES, Manuel Vaz. O motor de indução trifásico. Porto: DEEC,1994. p. 1-104.

INDÚSTRIA WEG. Oportunidades em eficiência energética. Disponível em http://www.weg.net/institutional/BR/pt/news/geral/oportunidades-em-eficiencia-energetica. Acesso em: 01 mar. 2018.

MINISTÉRIO DE MINAS E ENERGIA. MME abre consulta pública sobre Motores Elétricos Trifásicos. Disponível em http://www.mme.gov.br/web/guest/paginainicial/outras-noticas/-/asset_publisher/32hLrOzMKwWb/content/mme-abre-consultapublica-sobre-eficiencia-de-motores-eletricos-trifasicos. Acesso em: 15 jan. 2018.

VENERO, Danyde Moraes; HIYODO, Sérgio Akio. Gestão de reparo de motores elétricos com foco em confiabilidade e otimização de custos. $23^{\circ}$ Congresso Brasileiro de Manutenção, Santos, 2008.

\section{DADOS OS AUTORES}

\section{Nome: Vinicius Marcos Pinheiro}

E-mail: viniciusmarcospinheiro@hotmail.com

Currículo Lattes: http://lattes.cnpq.br/8748537162782615

Mestrando em Engenharia Elétrica e graduação em Engenharia Elétrica pela Universidade Federal de Uberlândia (UFU). Experiência na área de máquinas elétricas, acionamentos elétricos e controle e automação.

\section{Nome: Luciano Coutinho Gomes}

E-mail: lcgomes@ufu.br

Currículo Lattes: http://lattes.cnpq.br/3372531394380706 
PINHEIRO, Vinícius Marcos; GOMES, Luciano Coutinho; OLIVEIRA, Cássio Alves de. Plataforma experimental para análise do rendimento de motores de indução trifásicos por meio de ensaios específicos

Doutorado, mestrado e graduação em Engenharia Elétrica pela UFU. Atualmente é professor da Faculdade de Engenharia Elétrica da UFU. Experiência na área de Acionamentos Elétricos, Processamento Digital de Sinais, Máquinas Elétricas e Controle e Automação Industrial.

\section{Nome: Cássio Alves de Oliveira}

E-mail: kass-07@hotmail.com

Currículo Lattes: http://lattes.cnpq.br/6920155455622196

Doutorando e mestrado (linha de pesquisa: acionamentos de máquinas elétricas) em Engenharia Elétrica pela UFU e graduação em Engenharia Elétrica com certificação em Engenharia de Sistemas de Energia Elétrica pela UFU. Experiência na área de Máquinas Elétricas, Acionamentos Elétricos e Controle e Automação. 